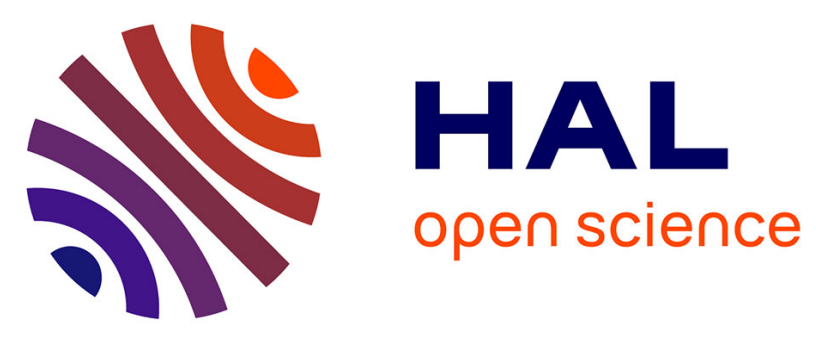

\title{
Réinvestigation de la structure des flagelles spermatiques: Cas particulier des spermatozoïdes à mitochondrie annulaire
}

Claudine Mattei, Xavier Mattei, Bernard Marchand, Roland Billard

\section{To cite this version:}

Claudine Mattei, Xavier Mattei, Bernard Marchand, Roland Billard. Réinvestigation de la structure des flagelles spermatiques: Cas particulier des spermatozoïdes à mitochondrie annulaire. Journal of Ultrastructure Research / Journal of Ultrastructural Research, 1981, 74 (3), pp.307-312. 10.1016/S00225320(81)80121-9 . hal-01600165

\section{HAL Id: hal-01600165 \\ https://hal.science/hal-01600165}

Submitted on 2 Jun 2020

HAL is a multi-disciplinary open access archive for the deposit and dissemination of scientific research documents, whether they are published or not. The documents may come from teaching and research institutions in France or abroad, or from public or private research centers.
L'archive ouverte pluridisciplinaire HAL, est destinée au dépôt et à la diffusion de documents scientifiques de niveau recherche, publiés ou non, émanant des établissements d'enseignement et de recherche français ou étrangers, des laboratoires publics ou privés.

\section{다(1)(2)}

Distributed under a Creative Commons Attribution - ShareAlikel 4.0 International 


\title{
Réinvestigation de la structure des flagelles spermatiques: Cas particulier des spermatozoïdes à mitochondrie annulaire
}

\author{
Claudine Mattei,* Xavier Mattei,* Bernard Marchand,* et Roland Billard $\dagger$ \\ *Département de Biologie Animale, Faculté des Sciences, Dakar, Sénégal, et \\ $\dagger$ Laboratoire de Physiologie des Poissons, INRA, 78350 Jouy-en-Josas, France
}

Received June 11, 1980, and in revised form January 8, 1981

\begin{abstract}
Among various teleost fishes studied in electron microscopy, five families show a spermatozoon with a middle piece only made of a ring-shaped mitochondrion. Each of them has a spermatic flagellum with a septum into the A tubule of some doublets. According to the family, doublets $1,2,5,6,7 ; 1,3,5,6,7$ or $1,2,3,5,6,7$ are septated. In literature, we have been looking for references to other animals having a spermatozoon with a ring-shaped mitochondrion and such a peculiar flagellum. Thus, we again found an intratubular septum into doublets $1,2,6$, 7 of a holeost fish spermatic flagellum, and also into doublets 1, 2, 5, 6, 7 of a holothurian spermatic flagellum. Nevertheless, this flagellar dissymmetry appears not to be closely related to the annular mitochondrion, but most likely to the general dissymmetry of the spermatozoon.
\end{abstract}

Dans un article précédent (Mattei et al., 1979) nous avons décrit une structure particulière associée aux doublets $\mathrm{n}^{0} 1,2,5$, et 6 de l'axonème chez des poissons téléostéens. Nous supposions l'existence d'une corrélation fonctionnelle entre cette dissymétrie particulière du flagelle et la dissymétrie générale du spermatozoïde correspondant.

Nous rendons compte ici des particularités de l'axonème des spermatozoïdes qui possèdent une mitochondrie annulaire unique.

\section{MATERIEL ET METHODES}

Des fragments de testicules mûrs ont été prélevés sur des poissons téléostéens appartenant aux espèces et familles suivantes: Sardinella aurita et Ethmalosa fimbriata (Clupeidae), Anchoa guineensis (Engraulidae), Xenodermichthys sp. (Alepocephalidae), Searsia sp. (Searsidae), et Salmo gairdneri (Salmonidae). Les Clupeidae et Engraulidae ont été récoltés au Sénégal à l'aide de senne de rivage. Les Alepocephalidae et Searsidae ont été pêchés le 12 avril 1975 au large des côtes sénégalaises par chalutage à $600 \mathrm{~m}$ de profondeur lors d'une campagne de pêche du bateau océanographique russe: Le Bielogorsk. Les Salmonidae, représentés par la truite "arc-en-ciel" proviennent de l'élevage du laboratoire de Jouy-en-Josas; les mâles adultes ont été sacrifiés en fin novembre et dé- but décembre, c'est-à-dire en début de spermiation. Des fragments de testicules sont préparés pour la microscopie électronique suivant les méthodes de routine précédemment décrites (Mattei et al., 1979). Les observations sont faites aux microscopes électroniques OPL MEU $100 \mathrm{kV}$ et Siemens Elmiskop 101.

\section{OBSERVATIONS}

Les spermatozoïdes que nous étudions possèdent un noyau arrondi ou ovoïde qui présente à sa base une dépression plus ou moins importante, pouvant constituer un véritable canal nucléaire dans lequel se place le diplosome (Fig. 1). Au niveau de la pièce intermédiaire, une mitochondrie unique annulaire entoure le début du flagelle (Figs. 1, 2, et 9). Les jeunes spermatides possèdent plusieurs mitochondries; celles-ci fusionnent par la suite en un seul élément sphérique qui, lors de la maturation du spermatozoïde, s'organise en un anneau périflagellaire.

\section{Le flagelle du spermatozoïde}

Les flagelles étudiés possèdent une structure classique de type $9+2$. Des bras sont associés au tubule $\mathrm{A}$ de chaque doublet. Les tubules A ne paraissent pas tous iden- 


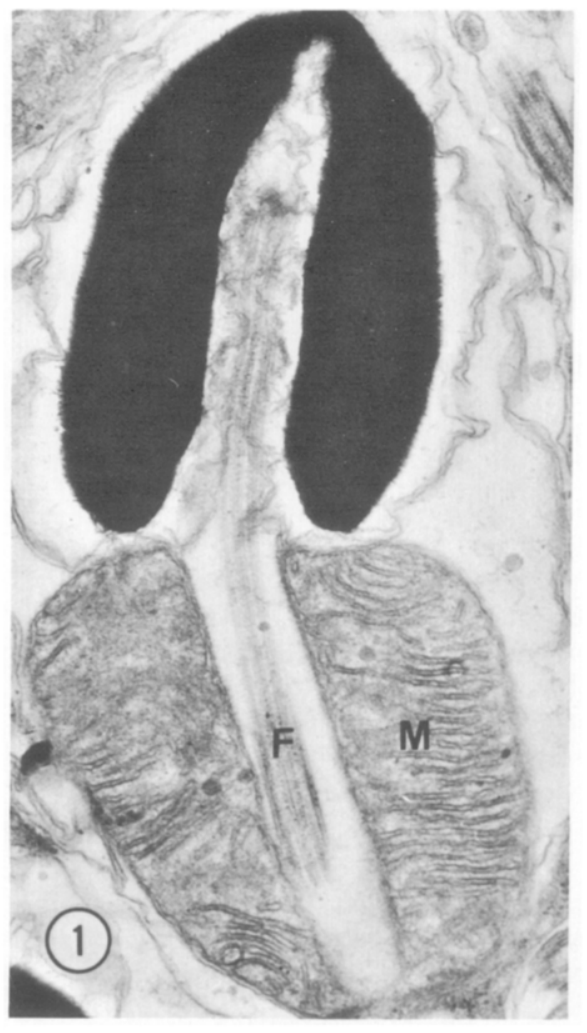

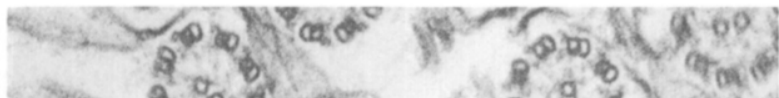

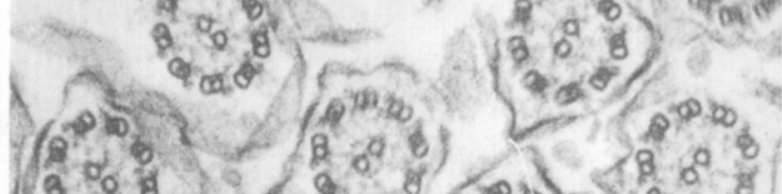

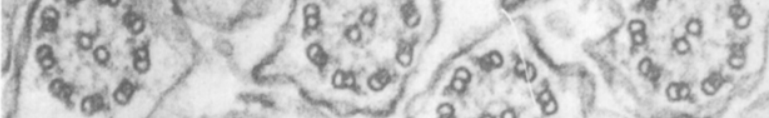

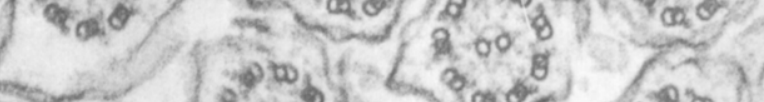

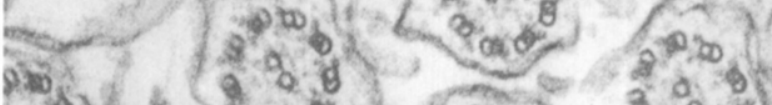

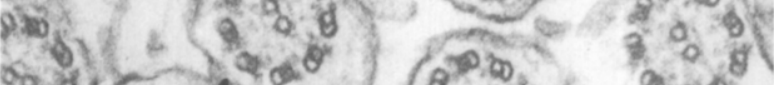

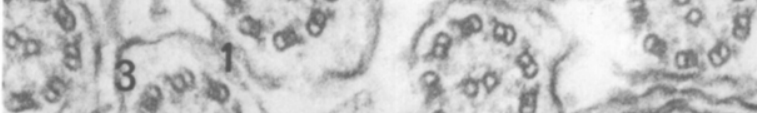

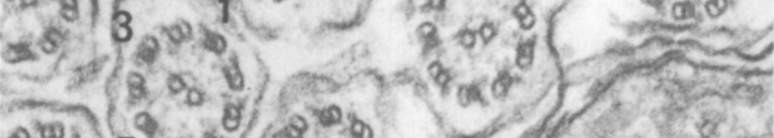

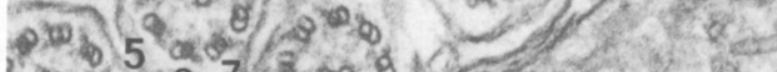
a. 679 , $608 \mathrm{~B}$ flet

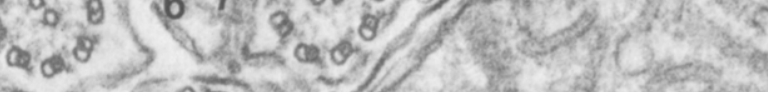

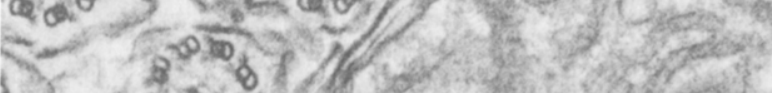

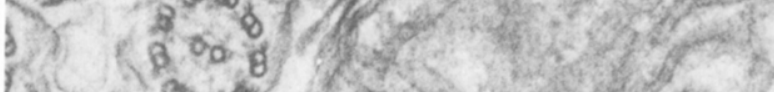
(2)

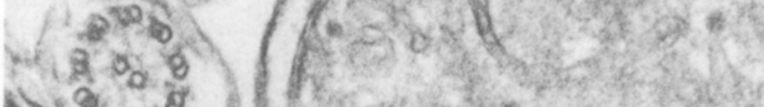

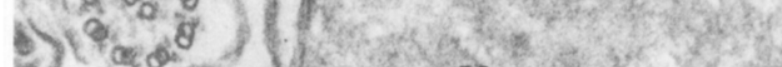

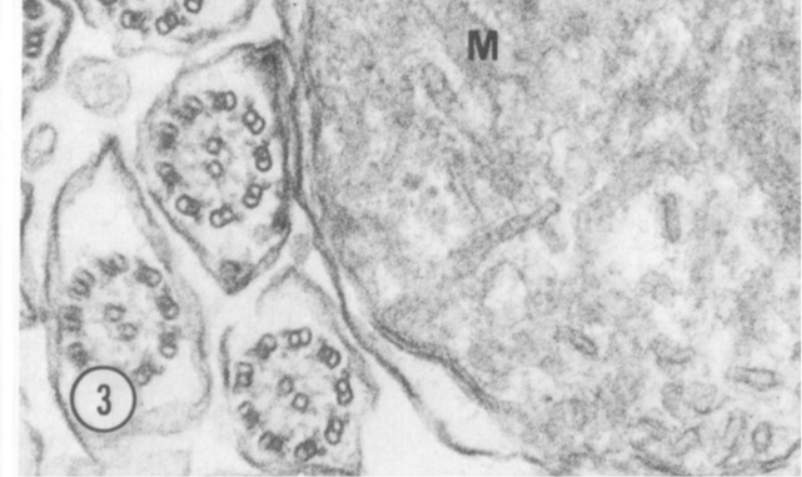
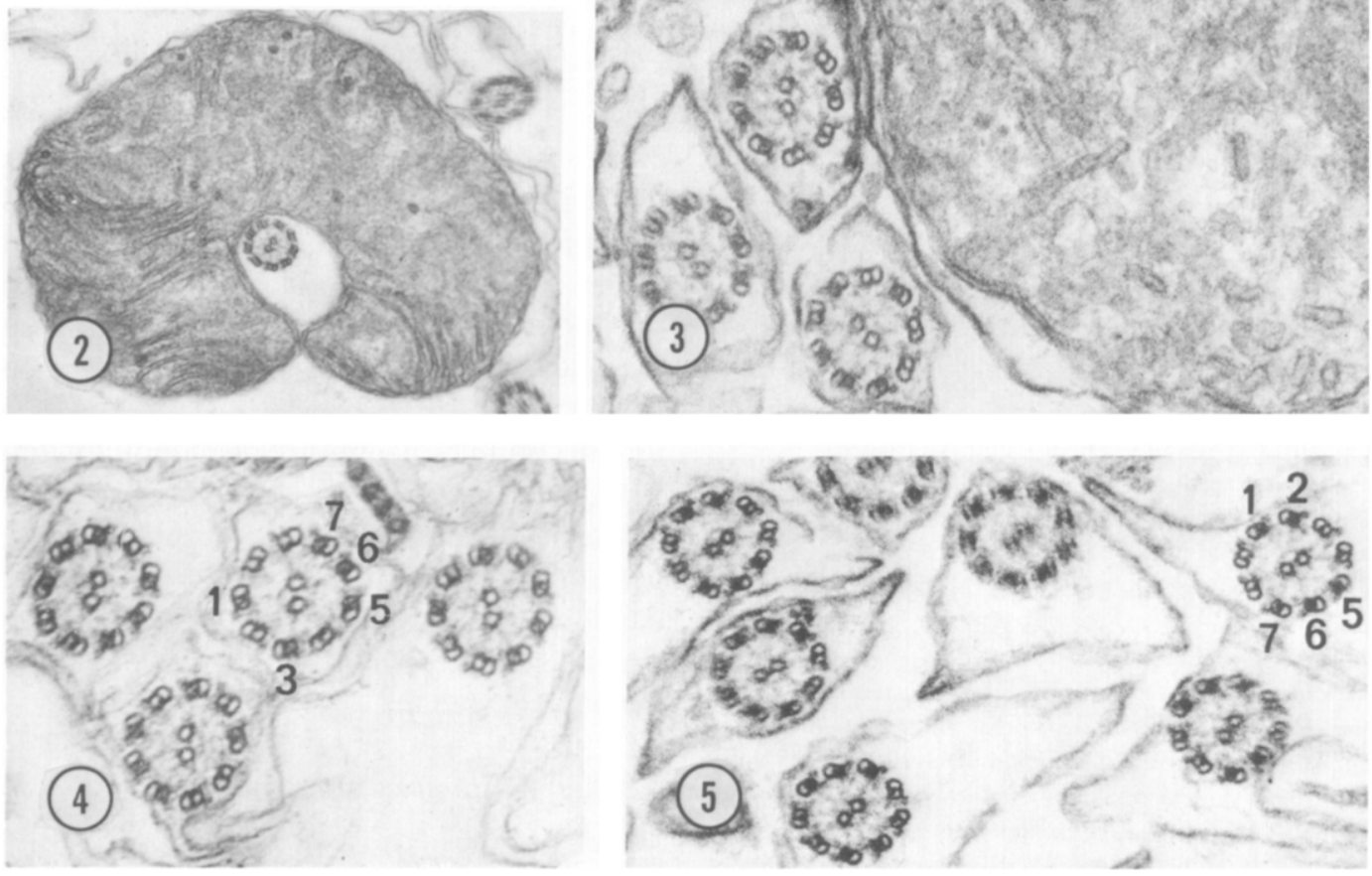
TABLEAU I

RÉPARTITION DES DOUBLETS MONTRANT UN CLOISONNEMENT DU TUBULE A

\begin{tabular}{|c|c|c|c|c|c|c|c|c|c|c|}
\hline \multirow{2}{*}{\multicolumn{2}{|c|}{ Genres étudiés }} & \multicolumn{9}{|c|}{ Numéro des doublets } \\
\hline & & 1 & 2 & 3 & 4 & 5 & 6 & 7 & 8 & 9 \\
\hline Echinoderme & Cucumaria & + & + & & & + & + & + & & \\
\hline Holostéen & Lepisosteus & + & + & & & & + & + & & \\
\hline \multirow[t]{6}{*}{ Téléostéen } & Searsia & + & + & & & + & + & + & & \\
\hline & Xenodermichthys & + & + & & & + & + & + & & \\
\hline & Salmo & + & + & + & & + & + & + & & \\
\hline & Sardinella & + & & + & & + & + & + & & \\
\hline & Ethmalosa & + & & + & & + & + & + & & \\
\hline & Anchoa & + & & + & & + & + & + & & \\
\hline
\end{tabular}

tiques; certains montrent une opacité plus importante aux électrons. Nous avons pu mettre en évidence que cette augmentation de la densité résultait de la présence d'une cloison traversant le tubule A dans le prolongement de son bras externe. Les tubules A qui présentent cette particularité sont toujours les mêmes pour une espèce donnée. Chez Anchoa guineensis, Sardinella aurita, et Ethmalosa fimbriata ce sont les doublets $\mathrm{n}^{0} 1,3,5$, 6 , et 7 qui sont cloisonnés (Figs. 3 et 4 ), chez Searsia sp. et Xenodermichthys sp. (Figs. 5 et 6), ce sont les doublets $\mathrm{n}^{\circ} 1,2,5,6$, et 7 , tandis que chez Salmo trutta ce sont les doublets $\mathrm{n}^{\circ}$ 1, 2, 3, 5, 6, et 7 (Fig. 7).

Dans la spermatide jeune le flagelle ne présente aucune cloison intratubulaire. Les doublets apparaissent alors vides sur toute leur longueur (Fig. 8). Les cloisons intratubulaires n'apparaissent qu'à la fin de la spermiogenèse.

Dans le spermatozoïde mûr ces cloisons ne sont pas observables sur toute la lon- gueur du flagelle; elle sont absentes au niveau de la pièce intermédiaire (Figs. 2 et 9) et de l'extrémité distale du flagelle. De plus, certaines coupes transversales de flagelles ne montrent qu'une partie des cinq ou six cloisons intratubulaires caractéristiques de l'espèce étudiée (Fig. 9). La fermeture de l'anneau mitochondrial ne semble pas se faire au hasard. Elle se situe le plus souvent en face du doublet $n^{\circ} 2$ (Figs. 2 et 9 ).

\section{DISCUSSION}

En 1959 Afzelius décrit des bras sur les doublets flagellaires, ce qui permet de numéroter avec précision les 9 doublets de l'axonème de type $9+2$. Nous employons ici cette numérotation. En 1960, Nagano, Gibbons, et Grimstone ainsi que Fawcett remarquent que les tubules A et B qui constituent les doublets périphériques des flagelles ne sont pas identiques. Le tubule A portant les bras contient un matériel dense aux électrons tandis que le tubule B est creux. Ceci a été observé par la suite

FIG. 1. Coupe Iongitudinale du spermatozoïde de Anchoa guineensis. Une mitochondrie (M) entoure le flagelle $(\mathrm{F})$ au niveau de la pièce intermédiaire. $\times 17000$.

FIG. 2. Coupe transversale du spermatozoïde de Anchoa guineensis passant au niveau de la pièce intermédiaire. Une mitochondrie unique constitue un manchon périflagellaire. $\times 23000$.

Fig. 3. Coupe transversale de flagelles de spermatozoïdes de Ethmalosa fimbriata. Des cloisons sont visibles dans les tubules A des doublets $1,3,5,6$, et 7 . M, mitochondrie. $\times 60000$.

FIG. 4. Coupe transversale de flagelles de spermatozoïdes de Anchoa guineensis. Des cloisons sont présentes dans les tubules $\mathrm{A}$ des doublets $1,3,5,6$, et $7 . \times 54000$.

Fıg. 5. Coupe transversale de flagelles de spermatozoïdes de Searsia sp. Les doublets 1, 2, 5, 6, et 7 montrent une cloison dans leur tubule A. $\times 50000$. 
MATTEI ET AL.
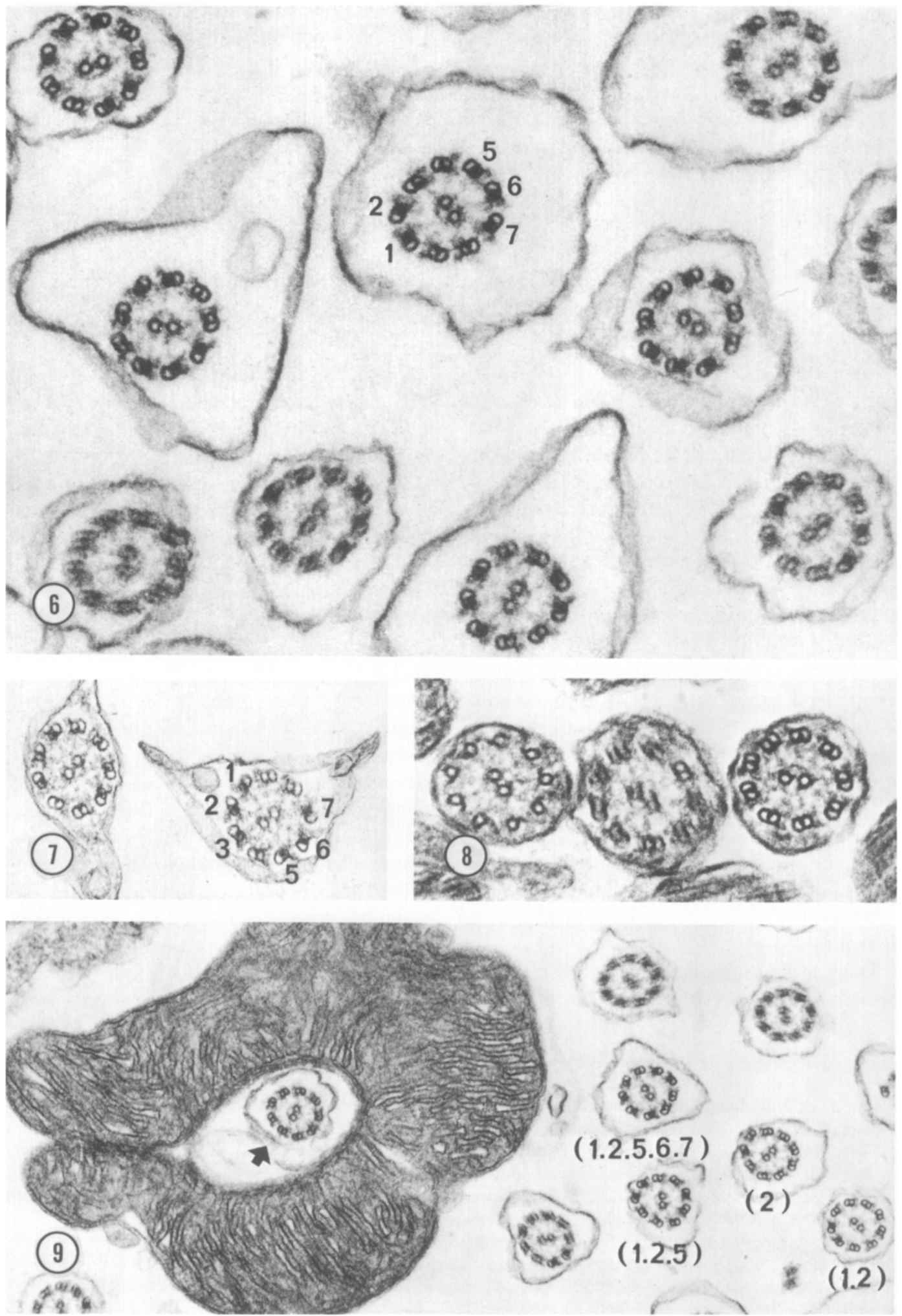
dans de nombreux cils et flagelles comme nous l'avons précédemment dit (Mattei et al., 1979). Certains auteurs ont remarqué que cette densité du tubule $\mathrm{A}$ était due à la présence d'une cloison traversant le tubule dans le prolongement du bras externe (Gibbons, 1961; Reese, 1965). Nous avons mentionné dans certains flagelles dont le spermatozoïde est dissymétrique (Mattei et al., 1979) que quatre doublets seulement $(1,2$, 5 , et 6) montraient ce cloisonnement. Nous retrouvons une autre fixité dans la distribution des cloisons intratubulaires dans des flagelles de spermatozoïdes variés mais qui présentent un caractère commun: leur pièce intermédiaire est constituée d'une mitochondrie annulaire. Suivant les familles la distribution des cloisons intratubulaires se fait dans des groupes différents de doublets: $1,2,5,6$, et $7 ; 1,3,5,6$, et 7 , et $1,2,3,5,6$, et 7 . Nous interprétons les sections de flagelles dépourvus de toute formation dans les tubules $\mathrm{A}$ ou n'en possèdant que sur quelques doublets comme des extrémités flagellaires, avec une interruption étagée des cloisons, ou bien une mise en place des cloisons dans un flagelle de spermatide âgée.

Notre étude se rapportant uniquement à des poissons téléostéens, nous avons recherché parmi les travaux d'autres auteurs, si cette singularité flagellaire n'était pas visible chez d'autres animaux au spermatozoïde également pourvu d'une mitochondrie annulaire. Nous avons ainsi remarqué chez un autre poisson du groupe des Ho- lostéens (Afzelius, 1978), la présence de tubules $\mathrm{A}$ denses, vraisemblablement dû à l'existence d'une cloison. L'auteur présente à la Fig. 9 trois coupes transversales de flagelles; pour deux d'entre elles les doublets intéressés sont les $\mathrm{n}^{\circ} 1,2,6,7$, et dans la troisième trois doublets seulement semblent cloisonnés: 1,2 , et 7 . Ce même auteur montre également à la Fig. 5 que ces formations sont absentes dans la région proche de la pièce intermédiaire. Chez un invertébré qui possède également un spermatozoïde à mitochondrie annulaire: $\mathrm{Cu}$ cumaria miniata, Fontaine et Lambert (1976) présentent deux coupes de flagelles à la Fig. 4 qui montrent bien des tubules $\mathrm{A}$ opaques aux électrons au niveau des doublets $1,2,5,6$, et 7 . En rassemblant nos observations ainsi que celles de ces auteurs, il est possible de dresser le Tableau I et de faire la constatation suivante: les doublets 4,8 , et 9 ne sont pas concernés tandis que les doublets 1,6 , et 7 sont toujours cloisonnés dans ce type de spermatozoïde.

Cette dissymétrie spermatique ne peut pas être liée uniquement à la présence d'une mitochondrie annulaire, mais doit l'être plus généralement à la dissymétrie d'ensemble du spermatozoïde. En effet, nous avons précédemment décrit des flagelles spermatiques présentant des cloisons intratubulaires au niveau de leur doublets 1, 2, 5, et 6 (Mattei et al., 1979). Mais dans ces derniers cas la pièce intermédiaire n'est pas constituée d'une seule mitochondrie

FIG. 6. Coupe transversale de flagelles spermatiques de Xenodermichthys sp. Les cloisons intratubulaires sont présentes dans les doublets $1,2,5,6$, et $7 . \times 90000$.

FIg. 7. Coupe transversale de flagelles spermatiques chez Salmo gairdneri. Des cloisons intratubulaires sont présentes dans les doublets $1,2,3,5,6$, et $7 . \times 50000$.

FIg. 8. Flagelles de jeunes spermatides de Sardinella eba. La section de gauche montre un flagelle dans lequel les tubules A sont en place et les tubules B en formation. La section de droite présente un flagelle dont les doublets sont constitués. Aucune cloison intratubulaire n'est visible. $\times 90000$.

FIG. 9. Coupe transversale de flagelles chez Xenodermichthys sp. Le flagelle est dépourvu de cloisons intratubulaires au niveau de la pièce intermédiaire (flèche). Les sections montrent des flagelles pourvus de cloisons sur certains doublets: un doublet $(2)$, deux doublets $(1,2)$, trois doublets $(1,2,5)$, et cinq doublets $(1$, $2,5,6,7) . \times 35000$. 
annulaire, mais de plusieurs mitochondries; en revanche l'organisation générale de ces spermatozoïdes est très dissymétrique.

\section{BIBLIOGRAPHIE}

Afzelius, B. A. (1959) J. Biophys. Biochem. Cytol. $5,269-278$.

AfZelius, B. A. (1978) J. Ultrastruct. Res. 64, 309_ 314.

FAwCETT, D. W. (1960) $10^{\circ}$ Congrès international de Biologie Cellulaire, p. 228, Paris.
Fontaine, A. R., et Lambert, P. (1976) J. Morphol. 148, 209-226.

GibBons, I. R. (1961) J. Biophys. Biochem. Cytol. 11, 179-205.

Gibions, I. R., et Grimstone, A. W. (1960) J. Biophys. Biochem. Cytol. 7, 697-715.

Mattei, C., Mattei, X., et Marchand, B. (1979) J. Ultrastruct. Res. 69, 371-377.

Mattei, X. (1970) en Baccetti, B. (Ed.), Comparative Spermatology, pp. 531-552, Academic Press, New York.

Nagano, T. (1960) J. Appl. Physiol. 31, 1844. REESE, T. S. (1965) J. Cell Biol. 25, 209-230. 